\title{
Taxonomic status of Euzonitis haroldi (Heyden, 1870) (Coleoptera: Meloidae) inferred from morphological and molecular data
}

\author{
MARINA ALCOBENDAS ${ }^{1,3}$, JOSÉ L. RUIZ ${ }^{2}$, CHIARA SETTANNI ${ }^{1}$ \& MARIO GARCÍA-PARÍS ${ }^{1}$ \\ ${ }^{\prime}$ Museo Nacional de Ciencias Naturales. CSIC. José Gutiérrez Abascal, 2. 28006, Madrid. España \\ ${ }^{2}$ Instituto de estudios Ceutíes, Paseo del Revellín, 30, Ap. 583, 50080 Ceuta. España \\ ${ }^{3}$ Corresponding author. E-mail: marina@mncn.csic.es, Phone: +34/914111328, Fax: +34/915645078
}

\begin{abstract}
The species Euzonitis haroldi (Heyden 1870) (Coleoptera: Meloidae; Nemognathinae) is an almost unknown taxon, sometimes treated as a variety of E. quadrimaculata (Pallas, 1773), originally described from Central Spain and only known from a few localities in Spain and Morocco. We found specimens of E. haroldi and E. quadrimaculata feeding together on flowers of Apiaceae in Central Spain. These specimens provided an excellent opportunity to properly assess the status of $E$. haroldi by analyzing and comparing morphological and molecular data of the two taxa. For the morphological study we reviewed a total of 227 specimens. We concluded that $E$. haroldi is genetically and morphologically identical, except for coloration, to E. quadrimaculata, and therefore E. haroldi should be treated as a junior synonym of E. quadrimaculata (syn. nov.)
\end{abstract}

Key words: Taxonomy, mtDNA, Cytochrome oxidase I, Morphology, Spain, Morocco, New synonymy

\section{Introduction}

Euzonitis Semenov 1893, within the tribe Nemognathini Lacordaire 1859 (Coleoptera: Meloidae: Nemognathinae), is represented by eighteen species distributed from Madeira, the Maghreb and the Iberian Peninsula, to central Asia as far as Mongolia (Bologna \& Pinto 2002). Most species of Euzonitis are localized in western Asia and southeastern Europe, with only three species occurring in the westernmost portions of the Palearctic Region (Escherich 1891, 1897; Semenov 1893; Bologna 1979, 1991, 1994a, 1994b; Bologna \& Pinto 2002). The systematics and phylogenetic relationships of this genus, as well as that of many other genera of Nemognathinae, are poorly known (Bologna \& Pinto 2001, 2002). The first instar larval morphology, recently described for Euzonitis rubida (Ménétriés, 1832) (Di Giulio \& Bologna, 2007), confirms its closeness to Zonitis Fabricius, 1775 as previously suggested by Bologna (1991). The current taxonomy of the genus Euzonitis is mostly based on elytral and pronotal pilosity, integument sculpture and coloration; a set of characters that show a relatively high degree of intraspecific variation in blister beetles (Bologna 1991).

Three species of Euzonitis have been consistently reported from the Iberian Peninsula (García-París \& Ruiz 2005): E. sexmaculata (Olivier 1789), E. quadrimaculata (Pallas 1773) and E. haroldi (Heyden 1870) (Graells 1853; Salvañá Comas 1870; Martínez Sáez 1873; Escherich 1890, 1891, 1897; Champion 1902; Górriz Muñoz 1902; Navás 1902; Rodríguez López-Neyra 1914; De la Fuente 1933; Pardo Alcaide 1952, 1956; Bologna 1991; Recalde et al. 2002; Pérez-Moreno et al. 2003). While the taxonomic status of the widely distributed E. sexmaculata and E. quadrimaculata, seems to be well established (Bologna 1991), there is controversy on the status of $E$. haroldi. This taxon, originally described as Zonitis haroldi Heyden 1870 from Central Spain (loc. typ.: "Casa de Campo" near Madrid, Heyden 1870: p. 150) and only known from a few localities 